\title{
Centrifugal Distortion Analysis of the Microwave and Millimeter Wave Spectra of Deuterated Ketenes ${ }^{1,2}$
}

\author{
László Nemes ${ }^{3}$ and Manfred Winnewisser \\ Physikalisch-Chemisches Institut, Justus Liebig-Universität Giessen \\ (Z. Naturforsch. 31 a, 272-282 [1976] ; received November 18, 1975)

\begin{abstract}
Measurements of $\mathrm{R}$ and $\mathrm{Q}$ branch a-type pure rotational transitions in the frequency range from $8 \mathrm{GHz}$ to $220 \mathrm{GHz}$ are reported for ketene- $\mathrm{d}_{1}$ and ketene- $\mathrm{d}_{2}$. The microwave and millimeter wave transitions were analysed in terms of Watson's reduced Hamiltonian, including the sextic terms $H_{K J}$ and $H_{J K}$. The values of the inertial defect and the $\tau$ defect obtained from the centrifugal distortion analysis are in accord with the planar model for the ketene molecule.
\end{abstract}

\section{Introduction}

The importance of ketene for synthetic work, coupled with the fact that it has a relatively simple and symmetric molecular structure, initiated spectroscopic investigations comparatively early. It was the subject of some of the earliest microwave studies ${ }^{1-3}$.

The analysis of the infrared vibrational spectra ${ }^{4}$ and the microwave studies of pure rotational transitions ${ }^{1-3,5}$ have given us information about the molecular geometry and confirmed the $\mathrm{C}_{2 \mathrm{v}}$ symmetry. Thus, relatively dependable rotational constants have been obtained for the $\mathrm{H}_{2}, \mathrm{HD}$ and $\mathrm{D}_{2}$ substituted species ${ }^{1-4}$. It has also been concluded on the basis of the vibrational and rotational spectra that the ketene molecule is planar. This conclusion came mostly from the symmetry properties of rotational and rotational-vibrational transitions. There has been only one paper, by Johns, Stone and Winnewisser ${ }^{6}$, in which an inertial defect value for $\mathrm{H}_{2}$ ketene could be derived from the combined analysis of infrared and microwave data.

In the present paper we attempt to fill some gaps in the knowledge about the deuterium substituted species. We have measured sufficient millimeter wave transitions to perform the centrifugal distortion analysis for both ketene- $\mathrm{d}_{1}$ and ketene- $\mathrm{d}_{2}$. These analyses have yielded, in addition to refined

1 This research was supported by funds from the Deutsche Forschungsgemeinschaft.

2 Presented in part at the "Third Colloquium on High Resolution Molecular Spectroscopy", Tours, France, September $17-21,1973$, as Paper J. 6.

3 Present address: Central Research Institute for Chemistry, Hungarian Academy of Sciences, H-1025 Budapest, Pusztaszeri ut 59-67, Hungary.

Reprint requests to Dr. M. Winnewisser, PhysikalischChemisches Institut, Justus Liebig-Universität, HeinrichBuff-Ring 58, D-6300 Giessen, Germany. rotational constants, the quartic and sextic centrifugal distortion constants, values of the inertial defect and the $\tau$ defect. Comparison of these inertial defect values with values calculated from a normal coordinate analysis supports strongly the planar model for the ketene molecular structure.

The frequency predictions of pure rotational transitions together with their calculated standard deviations provide a basis for a possible search for interstellar deuterated ketene molecules.

\section{Experimental Procedures}

Both deuterated ketenes were prepared using a modified Hurd-lamp ${ }^{7}$ by pyrolysing hexadeuteroacetone and partially deuterated acetone. In the process of producing ketene- $\mathrm{d}_{1}$, following the method reported by Moore and Pimentel ${ }^{4}$, both normal ketene and ketene- $\mathrm{d}_{2}$ are also synthetized. We have not attempted to separate these species since in the vibrational ground state studies their presence has caused no problems. The pyrolysis of acetone gives a number of by-products apart from methane, principally $\mathrm{CO}_{2}$ and ethylene. These molecules are, however, nonpolar and therefore their presence in the sample does not lead to disturbing lines in the rotational spectrum of the ketenes. However, in order to avoid pressure broadening of the ketene absorption lines a distillation procedure was applied to remove all of the heavy methane and $\mathrm{CO}_{2}$, and most of the heavy ethylene.

The measurements of centimeter wave lines between 8 and $18 \mathrm{GHz}$, and between 24 and $36 \mathrm{GHz}$ were carried out using a Hewlet-Packard 8460A Molecular Rotational Resonance spectrometer. A Stark modulation of frequency $33.33 \mathrm{kHz}$ and a two meter X-band Stark cell were employed.

The millimeter wave range rotational transitions were measured by means of a spectrometer having 
a 2.5 meter free space absorption cell. The sources of radiation were different OKI klystrons in the frequency range $28-45 \mathrm{GHz}$. By means of harmonic multiplication the whole range between 50 and $250 \mathrm{GHz}$ could be covered. In order to increase the precision of the measurements and the detectability of weak lines data acquisition utilizing a PDP-8/I computer was applied, as described by Winnewisser ${ }^{8}$. This system provides an enhanced signal-to-noise ratio by means of signal averaging and smoothing. Frequency determination is performed by the computer using a line-center calculation program ${ }^{9}$. The accuracy of these measurements is believed to be better than $\pm 20 \mathrm{kHz}$ for the stronger lines.

Sample pressure in the room temperature cell was in the range $10-50 \cdot 10^{-3}$ Torr.

\section{General Features of the Pure Rotational Spectra}

The microwave and millimeter wave rotational spectra of the deuterated ketene molecules show the structure expected for $a$-type transitions of slightly asymmetric prolate top molecules. Although deuteration introduces more asymmetry than is the case for normal ketene, the asymmetry parameters do not change significantly. The value of $x$ is -0.99722 , -0.99489 and -0.99144 for ketene- $\mathrm{h}_{2}$, ketene- $\mathrm{d}_{1}$ and ketene- $\mathrm{d}_{2}$ respectively.

The appearance of the pure rotational spectra is governed by the small asymmetry and the sizeable centrifugal distortion effects. The intensities basically follow a prolate symmetric top intensity distribution, which is further regulated by nuclear spin statistics. For ketene- $d_{2}$ the statistical weight of the anti-symmetric levels is half that of the symmetric levels, so that transitions originating from levels of even $K_{\mathrm{a}}$ have half the weight of those originating from odd $K_{\mathrm{a}}$ levels.

Figure 1 compares the $K_{\mathrm{a}}$ pattern obtained for the three isotopically substituted ketenes for the transition $J=9 \leftarrow 8$ (the data for ketene- $h_{2}$ were taken from the work of Johns, Stone and Winnewisser $^{6}$ ). Deviations from the rigid-rotor pattern are
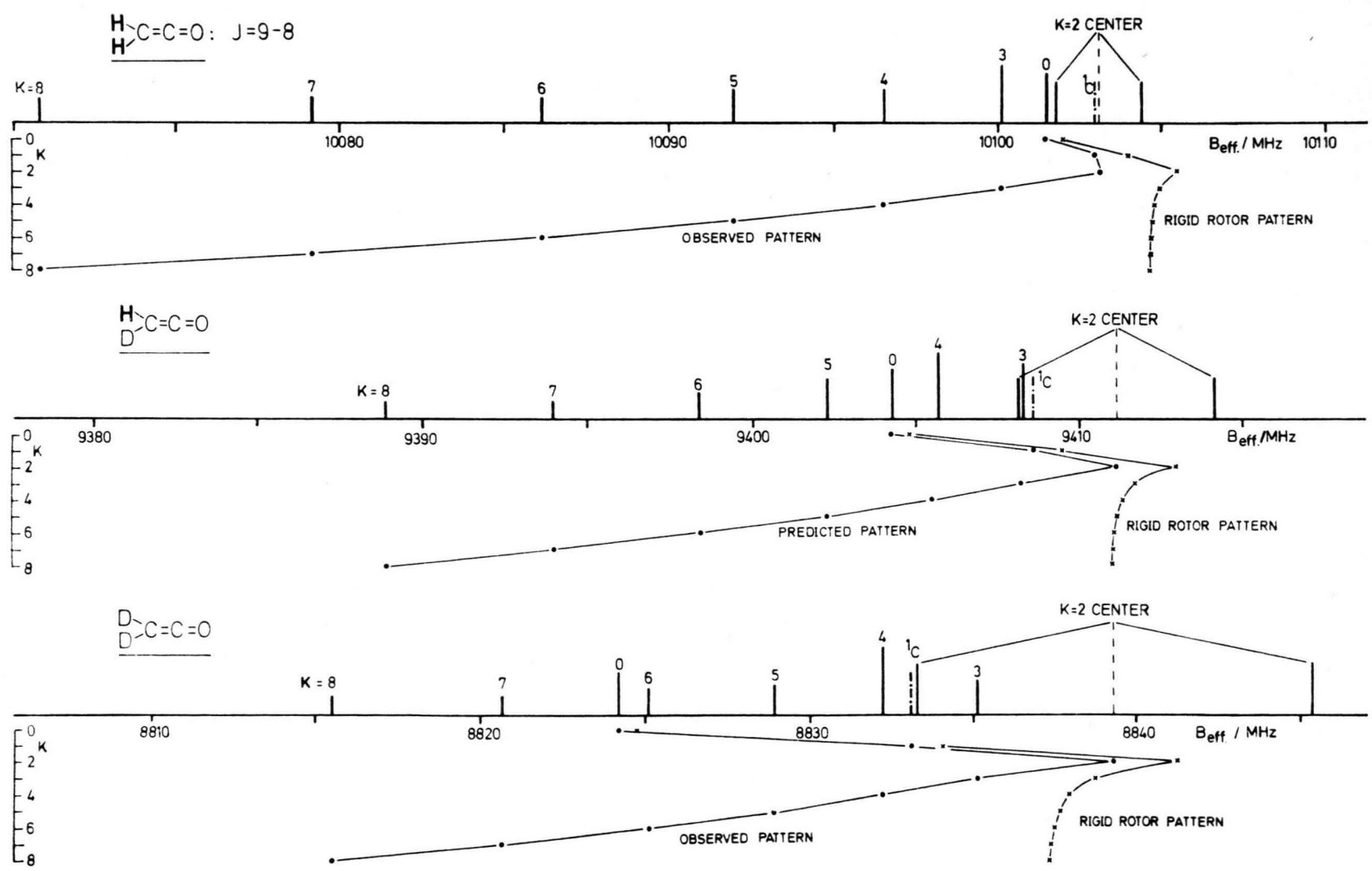

Fig. 1. The $K_{\mathrm{a}}$ patterns of typical $a$-type ${ }^{\mathrm{q}} \mathrm{R}$ branch pure rotational transitions for the ketene species. 


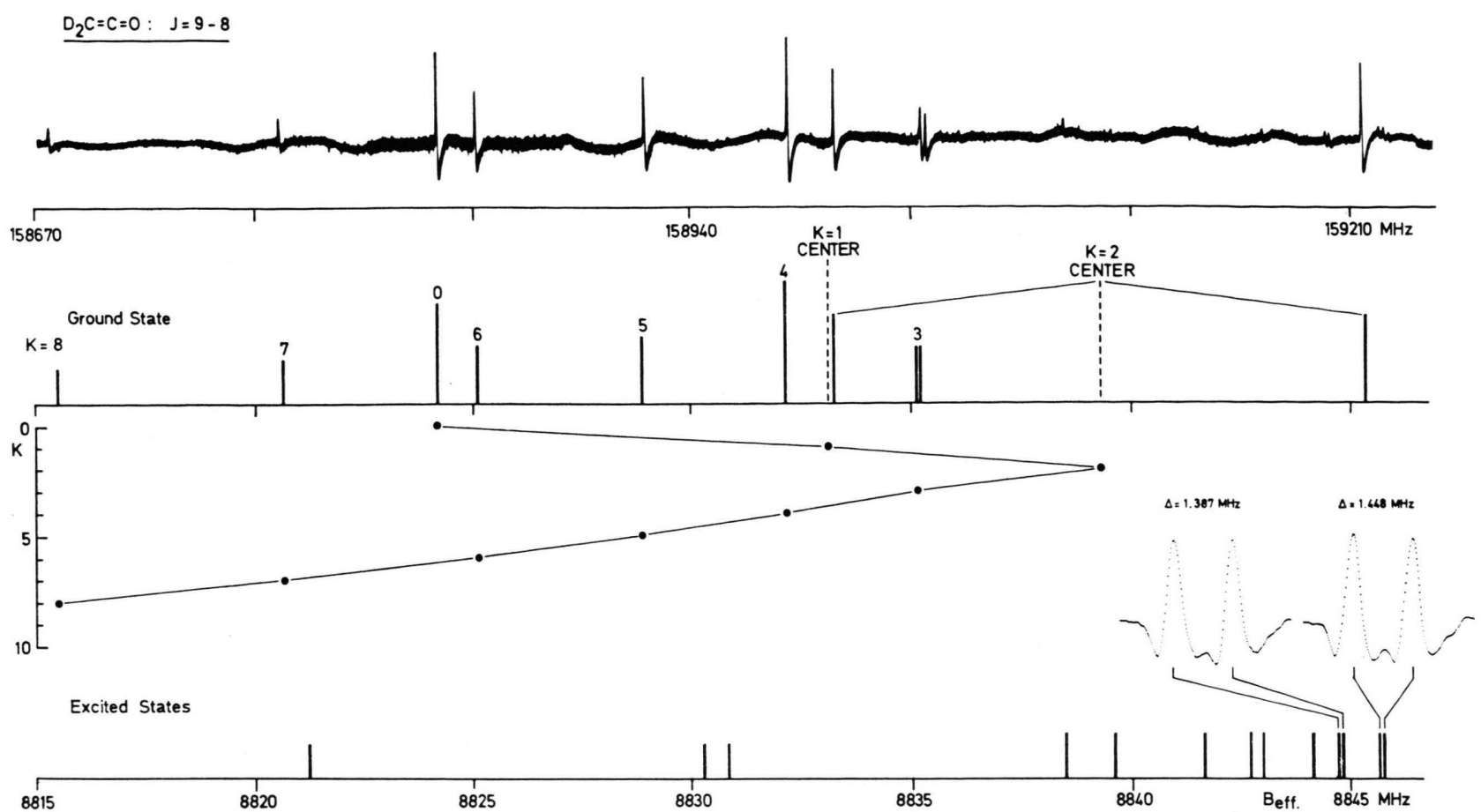

Fig. 2. Ground-state and excited state $a$-type ${ }^{\mathrm{q}} \mathrm{R}$ branch rotational transitions of $\mathrm{CD}_{2} \mathrm{CO}$. Both upper scale, giving the frequency of ground state lines, and lower scale giving $B^{\prime}$ eff $=v /(2 J+2)$ for excited state lines refer to the observed spectrum at the top of the Figure.

indicated. Thus it is seen that band heads are formed by the slight asymmetry, enhanced further by the centrifugal distortion effects. This enhancement is the smallest for ketene- $\mathrm{d}_{2}$ since it is the heaviest and least symmetric of the three species. The regularity of the $K_{\mathrm{a}}$ pattern proved to be a great help in the spectral assignments.

On-line signal averaging and smoothing made it possible to observe weak vibrational satellites belonging to the three lowest frequency vibrational states. Figure 2 depicts a typical R-branch transition and indicates a few lines originating from excited vibrational states. It was possible to observe the very small $K_{\mathrm{a}}=3$ asymmetry doubling in the ground vibrational state and also in the excited vibrational states. The inserts of Fig. 2 show a magnified view of these doublets as they actually appear on the screen of the oscilloscope. The analysis of these excited states will be published separately.

\section{Assignment of Rotational Transitions}

The approximate assignment of the rotational spectra presented no difficulties since rigid rotor predictions were available ${ }^{1,2,5,10}$. The number of a-type $\mathrm{R}$ branch transitions was 87 , while there were 18 a-type $Q$ branch transitions measured for ketene- $d_{2}$. For ketene- $d_{1} 49 \mathrm{R}$ branch transitions and $12 \mathrm{Q}$ branch transitions have been measured and used in the least-squares calculations.

Approximate centrifugal distortion parameters were at our disposal as preliminary results of recent normal-coordinate calculations ${ }^{11}$. These we used in a simplified centrifugal distortion program utilizing Polo's expansion formulas ${ }^{12}$, written for the PDP. 8/I computer.

Further refinement was done through a bootstrap procedure using an asymmetric rotor fitting program described by Johns and Olson ${ }^{13}$. The bootstrap method consisted in the first stage of fitting the observed lines to quartic distortion constants only. Later it was found necessary to include two higher-order, sextic distortion constants, $H_{J K}$ and $H_{K J}$, to remove most of the residual errors. A discussion of the trends in the residual errors is given in Section VI. 


\section{Treatment of Centrifugal Distortion Effects}

The centrifugal distortion analysis of the rotational spectra has been performed using Watson's reduced Hamiltonian operator formalism in the $I^{\mathrm{r}}$ - axis representation, constraining $R_{6}$ to zero. The form of the operator below indicates that, of the sextic distortion coefficients, only $H_{K J}$ and $H_{J K}$ have been determined in our calculations [for the complete form, see Eq. (37) in Ref. ${ }^{14}$ ] :

$$
\begin{aligned}
H_{\mathrm{red}}= & {\left[1 / 2(\tilde{B}+\tilde{C}) J^{2}+\{\tilde{A}-1 / 2(\tilde{B}+\tilde{C})\} J_{a}{ }^{2}-\Delta_{J}\left(J^{2}\right)^{2}-\Delta_{J K} J^{2} J_{a}^{2}-\Delta_{K} J_{a}^{4}+H_{J K}\left(J^{2}\right)^{2} J_{a}{ }^{2}+H_{K J} J^{2} J_{a}^{4}\right] } \\
& +\left[\left(J_{b}{ }^{2}-J_{c}{ }^{2}\right)\left\{1 / 4(\tilde{B}-\tilde{C})-\delta_{J} J^{2}-\delta_{K} J_{a}{ }^{2}\right\}+\left\{1 / 4(\tilde{B}-\tilde{C})-\delta_{J} J^{2}-\delta_{K} J_{a}^{2}\right\}\left(J_{b}{ }^{2}-J_{c}{ }^{2}\right)\right]
\end{aligned}
$$

where $J, J_{a}, J_{b}$ and $J_{c}$ are the operators for the total angular momentum and its components, and the square brackets indicate that the first group of operators is diagonal in $K$, while those in the second bracket are off-diagonal in $K$ by two units. The form of the second bracket also indicates that $J_{a}^{2}$ does not commute with $\left(J_{b}^{2}-J_{c}^{2}\right)$. The reduced rotational constants are given in Tables I and II for the two deuterated species of ketene.

Table I. Spectroscopic constants of $\mathrm{CD}_{2} \mathrm{CO}$ for Watson's reduced Hamiltonian in the $I^{r}$ axis representation a,

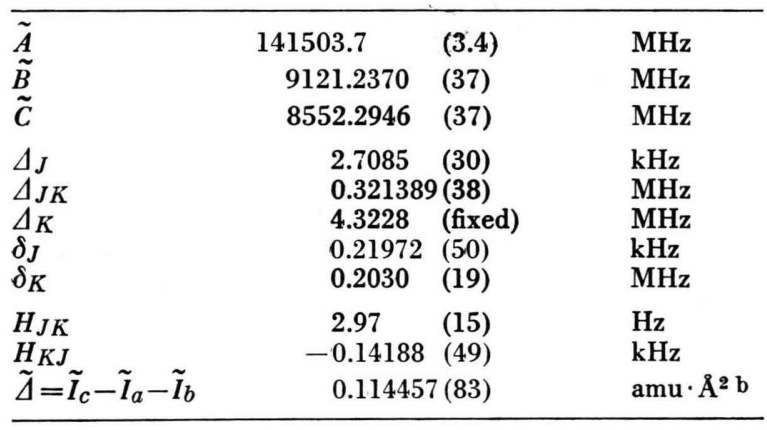

$\tilde{\varkappa}=-0.991441$

a The standard deviation of the fit is $19.7 \mathrm{kHz}$. Numbers in parentheses are standard errors.

b The value of the conversion constant;

const $=I_{b}\left(\mathrm{amu} \cdot \AA^{2}\right) \cdot B(\mathrm{MHz})$ used in this work is 505379 ; as calculated from the latest values of $h=6.626176(36)$. $10^{-27} \mathrm{erg} \cdot \mathrm{sec}$ and the Avogadro constant: $6.022045(31) \cdot$ $10^{23} \mathrm{~mol}^{-1}$ (Ref. ${ }^{24}$ ).

Since the difference $\left(A-\Delta_{K}\right)$ can be determined from $a$-type rotational transitions alone, but the two constants $A$ and $\Delta_{K}$ separately cannot, we have applied the following procedure. The value of $\Delta_{K}$ was first fixed at an estimate provided by normal-coordinate analysis utilizing the normal frequencies of all three isotopically substituted ketene species, their available centrifugal distortion constants and some $a$-type Coriolis zeta coefficients. The transitions were then fitted to the given Hamiltonian and a first approximation was obtained for the quartic
Table II. Spectroscopic constants of CHDCO for Watson's

\begin{tabular}{|c|c|c|c|}
\hline $\begin{array}{l}\tilde{A} \\
\tilde{B} \\
\tilde{C}\end{array}$ & $\begin{array}{r}194352.2 \\
9647.543 \\
9174.166\end{array}$ & $\begin{array}{l}(22.9) \\
(15) \\
(15)\end{array}$ & $\begin{array}{l}\mathrm{MHz} \\
\mathrm{MHz} \\
\mathrm{MHz}\end{array}$ \\
\hline $\begin{array}{l}\Delta_{J} \\
\Delta J_{K} \\
\Delta_{K} \\
\delta_{J} \\
\delta_{K}\end{array}$ & $\begin{array}{c}3.1637 \\
0.326970 \\
13.6709 \\
0.22525 \\
0.2392\end{array}$ & $\begin{array}{l}(67) \\
(77) \\
\text { (fixed) } \\
(92) \\
(74)\end{array}$ & $\begin{array}{l}\mathrm{kHz} \\
\mathrm{MHz} \\
\mathrm{MHz} \\
\mathrm{kHz} \\
\mathrm{MHz}\end{array}$ \\
\hline $\begin{array}{l}H_{J K} \\
H_{K J} \\
\tilde{\Delta}=\tilde{I}_{c}-\tilde{I}_{a}-\tilde{I}_{b}\end{array}$ & $\begin{array}{l}2.79 \\
-0.2779 \\
0.10264\end{array}$ & $\begin{array}{l}(40) \\
(14) \\
(30)\end{array}$ & $\begin{array}{l}\mathrm{Hz} \\
\mathrm{kHz} \\
\mathrm{amu} \cdot \AA^{2} \mathrm{~b}\end{array}$ \\
\hline
\end{tabular}
reduced Hamiltonian in the $I^{\mathrm{r}}$ axis representation a.

a The standard deviation of the fit is $23 \mathrm{kHz}$. Numbers in parentheses are standard errors.

b see footnote to Table I.

distortion constants. On the basis of these improved centrifugal distortion data another normal-coordinate analysis was made yielding now a modified $\Delta_{K}$-value. In the end two of the sextic distortion coefficients were also used in the refinement calculations. A comparison of our final, converged set of distortion constants to the normal-coordinate results is given in Tables III and IV.

In this way we have obtained better determined values for the $A$ rotational constant and for the

Table III. Comparison of least-squares values of quartic distortion coefficients of $\mathrm{CD}_{2} \mathrm{CO}$ to the results of normalcoordinate calculations $\mathrm{a}$.

\begin{tabular}{lllll}
\hline & L. S. & N.C. & \\
\hline$\Delta_{J}$ & 2.7085 & $(30)$ & 2.652 & $\mathrm{kHz}$ \\
$\Delta_{J K}$ & 0.321389 (38) & 0.33218 & $\mathrm{MHz}$ \\
$\Delta_{K}$ & 4.3228 (fixed) & 4.6103 & $\mathrm{MHz}$ \\
$\delta_{J}$ & 0.21972 & $(50)$ & 0.2194 & $\mathrm{kHz}$ \\
$\delta_{K}$ & 0.2030 (19) & 0.202 & $\mathrm{MHz}$ \\
\hline
\end{tabular}

a Although here the comparison is made between two sets corresponding to different values of $\Delta_{K}$, the differences of N.C. calcd. values corresponding to slightly different $\Delta_{K}$ are within the standard errors for the L. S. values. 
Table IV. Comparison of least-squares values of quartic distortion coefficients of CHDCO to the results of normalcoordinate calculations a.

\begin{tabular}{|c|c|c|c|}
\hline & L. S. & N. C. & \\
\hline$\Delta_{J}$ & 3.1637 & 3.160 & $\mathrm{kHz}$ \\
\hline$\Delta_{J K}$ & $0.326970(77)$ & 0.33138 & $\mathrm{MHz}$ \\
\hline$\Delta_{K}$ & 13.6709 (fixed) & 14.4657 & $\mathrm{MHz}$ \\
\hline$\delta_{J}$ & 0.22525 & 0.2283 & $\mathrm{kHz}$ \\
\hline$\delta_{K}$ & $0.2392 \quad(74)$ & 0.238 & $\mathrm{MHz}$ \\
\hline
\end{tabular}

a See footnote as for Table III.

Table V. Watsons's determinable spectroscopic constants for $\mathrm{CD}_{2} \mathrm{CO}[\mathrm{MHz}], \tau_{2}{ }^{*}=\tau_{2} /(\mathfrak{U}+\mathfrak{B}+\mathfrak{S})$.

\begin{tabular}{lccc}
\hline & & & $\begin{array}{l}\text { Norm. Coord. } \\
\text { calc. values } \\
\text { (Ref. 11) }\end{array}$ \\
\hline $\mathfrak{U}$ & 141503.7 & $(3.4)$ & \\
$\mathfrak{B}$ & 9121.1574 & $(37)$ & \\
$\mathfrak{E}$ & 8553.0279 & $(37)$ & -19.78069 \\
$\tau^{\prime}{ }_{a a a a}$ & -18.58759 & $(16)$ & -0.012365 \\
$\tau_{b b b b}^{\prime}$ & $-0.012592(16)$ & -0.008854 \\
$\tau^{\prime}{ }_{c c c c}$ & $-0.0090762(79)$ & \\
$\tau_{1}$ & -1.31806 & $(19)$ & \\
$\tau_{2}^{*}$ & $-0.0793027(77)$ & \\
$\Delta^{\prime} \tau$ & -0.000114 & $(25)$ & \\
\hline
\end{tabular}

inertia defect than would have been possible from just the $a$-type rotational transitions. A better method for solving this problem would, of course, be a simultaneous fit to some perpendicular ro-vibrational infrared transitions together with the $a$-type rotational transitions. No higher-resolution ro-vibrational data is, however, available to date on the deuterated ketene species.
Table VI. Watson's determinable spectroscopic constants for CHDCO $[\mathrm{MHz}], \tau_{2}{ }^{*}=\tau_{2} /(\mathfrak{U}+\mathfrak{B}+\mathfrak{E})$.

\begin{tabular}{lccc}
\hline & & & $\begin{array}{l}\text { Norm. Coord. } \\
\text { calc. values } \\
\text { (Ref. 11) }\end{array}$ \\
\hline $\mathfrak{U}$ & 194352.2 & $(22.9)$ & \\
$\mathfrak{B}$ & 9647.398 & $(15)$ & \\
$\mathfrak{E}$ & 9174.978 & $(15)$ & -59.20114 \\
$\tau^{\prime}{ }_{\text {aaaa }}$ & $-56.00414(34)$ & -0.014464 \\
$\tau^{\prime}{ }_{b b b b}$ & $-0.014457(34)$ & -0.010811 \\
$\tau^{\prime}{ }_{c c c c}$ & $-0.010853(19)$ & \\
$\tau_{1}{ }_{\tau^{*}}$ & $-1.34585(39)$ & \\
$\Delta^{*} \tau$ & $-0.068273(31)$ & \\
\hline
\end{tabular}

\section{Discussion}

\section{a) Discussion of the Results of Centrifugal Distortion Analysis}

It is possible to transform the spectroscopic constants obtained as Watson's determinable combinations of the Kivelson-Wilson quartic coefficients to calculate the so-called derived spectroscopic constants: $A, B, C, \tau_{a a b b}^{\prime}, \hbar^{4} \tau_{a b a b}, \tau_{b b c c}^{\prime}$ and $\tau_{c c a a}^{\prime}{ }^{14}$. This transformation involves the use of planarity conditions, rigorously valid only for equilibrium values of the quartic distortion coefficients. These planarity conditions were originally given by Dowling ${ }^{15}$, and by Oka and Morino ${ }^{16,17}$. We shall use these and similar planarity constraints following Kirchhoff ${ }^{18}$ and Cook et al. ${ }^{19,}{ }^{20}$ to provide several alternative sets of derived parameters. The following treatment is outlined in a paper by Yamada and Winnewisser ${ }^{21}$ and the present results are contained in Tables VII and VIII. The equations following

Table VII. Spectroscopic constants derived from determinable parameters via different planarity conditions for $\mathrm{CD}_{2} \mathrm{C} O \mathrm{O}$.

\begin{tabular}{|c|c|c|c|c|c|c|c|}
\hline Version & 1 & 2 & 3 & 4 & 5 & \multicolumn{2}{|c|}{$\begin{array}{l}\text { Norm. Coord. } \\
\text { calc. (Ref. 11) }\end{array}$} \\
\hline$A$ & $141503.7(3.4)$ & $141503.7(3.4)$ & $141503.7(3.4)$ & $141503.7(3.4)$ & $141503.7(3.4)$ & & $\mathrm{MHz}$ \\
\hline$B$ & $9121.1855(37)$ & $9121.1855(38)$ & 9121.2045 (37) & $9121.1855(37)$ & 9121.1951 (37) & & $\mathrm{MHz}$ \\
\hline$C$ & $8552.3460(37)$ & $8552.3473(38)$ & $8552.3270(37)$ & $8552.3459(37)$ & $8552.3364(37)$ & & $\mathrm{MHz}$ \\
\hline$I_{A}$ & $3.571489(85)$ & $3.571489(85)$ & $3.571489(85)$ & $3.571489(85)$ & $3.571489(85)$ & & $\mathrm{amu} \cdot \AA^{2}$ \\
\hline$I_{B}$ & $55.407162(25)$ & $55.407162(26)$ & $55.407046(24)$ & $55.407161(25)$ & $55.407103(25)$ & & $\mathrm{amu} \cdot \AA^{2}$ \\
\hline$I_{C}$ & $59.092441(25)$ & $59.092432(26)$ & $59.092572(24)$ & $59.092441(25)$ & $59.092507(25)$ & & $\mathrm{amu} \cdot \AA^{2}$ \\
\hline$\Delta$ & $0.113790(38)$ & $0.113781(37)$ & $0.114036(82)$ & $0.113790(38)$ & $0.113915(60)$ & & $\mathrm{amu} \cdot \AA^{2}$ \\
\hline$\tau_{a a b b}^{\prime}$ & $-1.36380(78)$ & $-1.36128(34)$ & $-1.4017(75)$ & $-1.36396(81)$ & $-1.3831(43)$ & & $\mathrm{MHz}$ \\
\hline$\tau^{\prime} b b c c$ & $-0.010556(11)$ & $-0.010556(11)$ & $-0.010556(11)$ & $-0.010394(17)$ & $-0.0104760(26)$ & -0.010317 & $\mathrm{MHz}$ \\
\hline$\tau_{c c a a}^{\prime}$ & $0.05630(61)$ & $0.05630(60)$ & $0.0942(73)$ & $0.05630(60)$ & $0.0755(40)$ & 0.0649 & $\mathrm{MHz}$ \\
\hline$\hbar^{4} \tau_{a a b b}$ & $0.14125(69)$ & $0.14125(68)$ & $0.14125(68)$ & $0.14125(69)$ & $0.1631(45)$ & 0.1572 & $\mathrm{MHz}$ \\
\hline$\hbar^{4} \tau_{a b a b}$ & $-0.75253(73)$ & $-0.75127(51)$ & $-0.7715(41)$ & $-0.75261(75)$ & $-0.7731(44)$ & -0.7861 & $\mathrm{MHz}$ \\
\hline$R_{6} \times 10^{6}$ & -8.69 & -8.69 & -8.69 & -13.8 & -11.2 & & $\mathrm{MHz}$ \\
\hline
\end{tabular}

a see footnote to Table I. 
Table VIII. Spectroscopic constants derived from determinable parameters via different planarity conditions for CHDCO a

\begin{tabular}{|c|c|c|c|c|c|c|c|}
\hline Version & 1 & 2 & 3 & 4 & 5 & \multicolumn{2}{|c|}{$\begin{array}{l}\text { Norm. Coord. } \\
\text { calc. (Ref. 11) }\end{array}$} \\
\hline$A$ & $194352.2(22.9)$ & $194352.2(22.9)$ & $194352.2(22.9)$ & $194352.2(22.9)$ & $194352.2(22.9)$ & & $\mathrm{MHz}$ \\
\hline$B$ & $9647.476(14)$ & $9647.476(15)$ & $9647.490(15)$ & $9647.476(15)$ & $9647.483(15)$ & & $\mathrm{MHz}$ \\
\hline$C$ & $9174.233(14)$ & $9174.234(15)$ & $9174.219(15)$ & $9174.233(15)$ & $9174.226(15)$ & & $\mathrm{MHz}$ \\
\hline$I_{A}$ & $2.60033(31)$ & $2.60033(31)$ & $2.60033(31)$ & $2.60033(31)$ & $2.60033(31)$ & & $\mathrm{amu} \cdot \AA^{2}$ \\
\hline$I_{B}$ & $52.384582(88)$ & $52.384582(91)$ & $52.384504(85)$ & $52.384582(88)$ & $52.384542(86)$ & & $\mathrm{amu} \cdot \AA^{2}$ \\
\hline$I_{C}$ & $55.086785(88)$ & $55.086780(91)$ & $55.086871(85)$ & $55.086785(88)$ & $55.086829(86)$ & & $\mathrm{amu} \cdot \AA^{2}$ \\
\hline$\Delta$ & $0.10188(14)$ & $0.10187(14)$ & $0.10204(30)$ & $0.10188(14)$ & $0.10196(22)$ & & $\mathrm{amu} \cdot \AA^{2}$ \\
\hline$\tau_{a a b b}^{\prime}$ & $-1.4895(24)$ & $-1.48800(96)$ & $-1.518(30)$ & $-1.4896(24)$ & $-1.504(16)$ & & $\mathrm{MHz}$ \\
\hline$\tau_{b b c c}^{\prime}$ & $-0.012384(26)$ & $-0.012384(26)$ & $-0.012384(26)$ & $-0.012310(44)$ & $-0.0123477(80)$ & -0.012350 & $\mathrm{MHz}$ \\
\hline $\boldsymbol{\tau}_{c c a a}^{\prime}$ & $0.1560(20)$ & $0.1560(20)$ & $0.185(29)$ & $0.1560(20)$ & $0.171(16)$ & 0.1711 & $\mathrm{MHz}$ \\
\hline$\hbar^{4} \tau_{a a b b}$ & $0.3105(22)$ & $0.3105(22)$ & $0.3105(22)$ & $0.3105(22)$ & $0.327(18)$ & 0.3372 & $\mathrm{MHz}$ \\
\hline$\hbar^{4} \tau_{a b a b}$ & $-0.9000(23)$ & $-0.8992(16)$ & $-0.914(16)$ & $-0.9000(23)$ & $-0.915(17)$ & -0.9297 & $\mathrm{MHz}$ \\
\hline$R_{6} \times 10^{6}$ & -8.47 & -8.47 & -8.47 & -10.8 & -9.6 & & $\mathrm{MHz}$ \\
\hline$S_{111} \times 10^{8}$ & 7.18 & 7.18 & 7.18 & 9.1 & 8.1 & & \\
\hline
\end{tabular}

a see footnote to Table I.

from the planarity conditions are summarized here for the different versions 1 through 5 (see also Yamada and Winnewisser ${ }^{21}$ ).

\section{Version 1}

These planarity equations are obtainable from those of Dowling ${ }^{15}$ and have the following form:

$$
\begin{aligned}
\hbar^{4} \tau_{a a b b} & =1 / 2\left[-\frac{B^{2}}{A^{2}} \tau_{a a a a}^{\prime}-\frac{A^{2}}{B^{2}} \tau_{b b b b}^{\prime}+\frac{A^{2} B^{2}}{C^{4}} \tau_{c c c c}^{\prime}\right], \\
\tau_{b b c c}^{\prime} & =1 / 2\left[-\frac{B^{2} C^{2}}{A^{4}} \tau_{a a a a}^{\prime}+\frac{C^{2}}{B^{2}} \tau_{b b b b}^{\prime}+\frac{B^{2}}{C^{2}} \tau_{c c c c}^{\prime}\right], \\
\tau^{\prime}{ }_{a a c c} & =1 / 2\left[+\frac{C^{2}}{A^{2}} \tau^{\prime}{ }_{a a a a}-\frac{A^{2} C^{2}}{B^{4}} \tau_{b b b b}^{\prime}+\frac{A^{2}}{C^{2}} \tau_{c c c c}^{\prime}\right] .
\end{aligned}
$$

In terms of these quantities the rest of the derived constants are calculated from:

$$
\begin{aligned}
\hbar^{4} \tau_{a b a b} & =\left[\tau_{1}-\left(\hbar^{4} \tau_{a a b b}+\tau_{b b c c}^{\prime}+\tau_{a a c c}^{\prime}\right)\right] / 2, \\
\tau_{a a b b}^{\prime} & =\hbar^{4}\left(\tau_{a a b b}+2 \tau_{a b a b}\right),
\end{aligned}
$$

and the $\tau$ defect, as derived from Eq. (67) in Watson's paper ${ }^{14}$ is :

$$
\Delta \tau=\tau_{c c c c}^{\prime}-\left(\tau_{2}-C \tau_{1}\right) /(A+B) .
$$

The $\tau$ defect is a rather sensitive function of $\tau_{2}$. $\tau_{1}$ and $\tau_{2}$ were calculated according to

and

$$
\tau_{1}=\tau_{a a b b}^{\prime}+\tau_{b b c c}^{\prime}+\tau_{a a c c}^{\prime}
$$

$$
\begin{aligned}
\tau_{2}= & -2(2 A-B-C) \Delta_{J}+4(B-C)\left(\delta_{J}+\delta_{K}\right) \\
& -2(B+C)\left(\Delta_{J K}+3 \Delta_{J}\right),
\end{aligned}
$$

which is identical to the equation for $\tau_{2}$ in Cook, De Lucia and Helminger ${ }^{22}$ (see Table VI there).

\section{Version 2}

The quantities $\hbar^{4} \tau_{a a b b}, \tau^{\prime}{ }_{b b c c}$ and $\tau^{\prime}{ }_{a a c c}$ are as in Version 1, but:

$\hbar^{4} \tau_{a b a b}=\left\{\tau_{2}-\left(A{\tau^{\prime}}_{b b c c}+B{\tau^{\prime}}_{a a c c}+C \hbar^{4} \tau_{a a b b}\right)\right\} / 2 C$.

Version 3

The quantities $\hbar^{4} \tau_{a a b b}, \tau^{\prime}{ }_{b b c c}$ and $\hbar^{4} \tau_{a b a b}$ are as in Version 1 and 2, while $\tau_{\text {aacc }}^{\prime}$ is given by

$\tau_{a a c c}^{\prime}=\left\{\tau_{c c c c}^{\prime}-\Delta \tau-\tau_{b b c c}^{\prime}(A-C) /(A+B)\right\} \frac{A+B}{B-C}$.

Version 4

The quantities $\hbar^{4} \tau_{a a b b}$ and $\tau_{c c a a}^{\prime}$ and $\hbar^{4} \tau_{a b a b}$ are as in Version 1, but:

$$
{\tau^{\prime}}_{b b c c}=\left\{\tau^{\prime}{ }_{c c c c}-\Delta \tau-\tau_{a a c c}^{\prime}\left(\frac{B-C}{A+B}\right)\right\} \frac{A+B}{A-C} .
$$

\section{Version 5}

Here $\hbar^{4} \tau_{a b a b}$ is calculated as in Version 1, while the rest of the quartic constants are obtained from:

$$
\begin{aligned}
& \hbar^{4} \tau_{a a b b}= \\
& {\left[-\left(\tau_{1}-\tau_{2} / C\right) / C-\frac{A-C}{B^{2}} \tau_{b b b b}^{\prime}-\frac{B-C}{A^{2}} \tau_{a a a a}^{\prime}\right] /} \\
& \left(\frac{A-C}{A^{2}}+\frac{B-C}{B^{2}}\right), \\
& \tau_{b b c c}^{\prime}=C^{2}\left\{\frac{\hbar^{4} \tau_{a a b b}}{A^{2}}+\frac{\tau_{b b b b}^{\prime}}{B^{2}}\right\}, \\
& \tau^{\prime}{ }_{a a c c}=C^{2}\left\{\frac{\tau_{a a a a}^{\prime}}{A^{2}}+\frac{\hbar^{4} \tau_{a a b b}}{B^{2}}\right\} .
\end{aligned}
$$


TABLE IK. OASERVFO AVJ CALCULATEJ FREQUENCIES OF JO:OO IN MHZ

\begin{tabular}{|c|c|c|c|c|c|c|c|c|}
\hline \multicolumn{2}{|c|}{ TRANSITION } & OBSERVE ก & CALCULATEO FREQUENCY & JBS.-CALC. & ENERGY & LEVELS & IN $C M-1$ & REMAYKS \\
\hline $\begin{array}{l}\text { UPPER } \\
\text { STATE }\end{array}$ & $\begin{array}{l}\text { LOWER } \\
\text { STATE }\end{array}$ & $\begin{array}{l}\text { FRE OUENCY } \\
\text { (WEIGHT) }\end{array}$ & (STANDARD DEVIATION) & & $\begin{array}{l}\text { UPPER } \\
\text { STATE }\end{array}$ & & $\begin{array}{l}\text { LOWER } \\
\text { STATE }\end{array}$ & \\
\hline
\end{tabular}

* A TYPE 2 BRANCH *

$1(0,1)-0(0,0)$

$2(0,2)-1(0,1)$ $2(1,1)=1(1,0)$

$3(0,3)=2(0,2)$ $3(1,2)=2(1,1)$ $3(1,3)-2(1,2$ $3(2,1)=2(2,0)$

$4(0,4)-3(0,3)$ $4(1,4)-3(1,3)$ $4(2,2)-3(2,1)$ $4(2,3)=3(2,2)$ $4(3,1)=3(3,0)$ $4(3,2)=3(3,0)$

$5(0,5)-4(0,4)$ $5(1,4)-4(1,3)$ $5(1,5)=4(1,4)$ $5(2,4)-4(2,3)$ $5(3,2)=4(3,1)$ $5(4,1)=4(4,0)$

$6(0,6)-5(0,5)$ $6(1,5)=5(1,4)$ $6(2,4)-5(2,3)$ $6(2,5)-5(2,4)$ $5(3,3)-5(3,2)$ $5(3,4)-5(3,3)$ $5(4,2)-5(4,1)$ $6(4,3)-5(4,2)$ $5(5,1)-5(5,0)$ $5(5,2)=5(5,1)$ $7(\pi, 7)-6(0,6)$ $7(1,7)=6(1,5)$ $7(1,7)=6(1,6)$ $7(2,6)=6(2,5)$ $7(3,4)=6(2,5)$ $7(3,5)-6(3,4)$ $(4,3)-6(4,2)$ $7(4,4)-6(4,3)$ $7(5,3)-6(5,2)$ $7(6,1)-6(6,0)$

(6, $21=6(6,1)$

$8(1,7)=7(1 ; 6)$ $9(1,8)-7(1,7)$ $8(2,6)=7(2,5)$ Q $(2,7)-7(2,6)$ $8(3,5)=7(3,4)$ $8(3,5)=7(3,5$ $9(4,4)=7(4,3)$ $8(4,5)-7(4,4)$ $8(5,3)=7(5,2)$ $8(6,2)=7(6,1)$ $3(5,3)=7(6,2)$ B $(7,1)=7(7,0)$ $8(7,2)=7(7,1)$

$9(0,9)-8(\llcorner, 8)$ $9(1,8)=8(1,7)$ $9(1,9)=8(1,8)$ $9(2,7)=8(2,6)$ $9(3,6)=8(3,5)$ $9(3,7)-8(3,6)$ $9(4,5)-8(4,4)$ $9(4,6)-8(4,5)$ $9(5,4)-8(5,3)$ $9(5,5)-9(5,4$ $9(6,3)-8(6,2$ $9(7,2)=8(7,1)$ $9(7,3)=8(7,2)$ ( 8,1$)=8(8,0)$ $9(8,2)-8(8,1)$

$10(0,10)-9(0,9)$ $10(1,9)-9(1,8$ $10(1,10)=9(1,9)$
$10(2,8)=9(2,7)$ $10(2,9)-9(2,8$ $10(3,7)-9(3,5$ $10(3,8)-9(3,7)$ $10(4,6)=9(4,5)$ $10(4,7)=9(4,6)$ $10(5,5)-9(5,4)$ $10(5,6)=9(5,5)$ $1016,5)=9(6,4)$ $10(7,3)=9(7,2)$ $10(7,4)-9(7,3)$ $10(8,2)-9(8,1)$ $10(8,3)-9(8,2)$
$17673.5723(1.0)$

$35345.1623(1.0)$ $35913.8193(1.0)$ $34777.5713(1.0)$

$53013.0360(1.0$ $53869.4143(1.0)$
$52165.0500(1.0)$ $53019.9460(1.0)$ $53012.5830(1.0)$

$70675.2453(1.0)$ $71823.4592(1.0)$ $60551.0648(1.0)$ $70699.9342(1.3)$

$76681.7524(1.0)$

$7 i 673.9173(1.5)$

$88329.9365(1.0)$ $89775.3875(1.0)$ $86935 . ; 158(1.0)$ $88385.3603(1.2)$ 88349.465511 .01 $88343.507 \geqslant(0.0)$ $88343.507 ?(1.0)$ $88319.4834(1.0)$

$105975.1769(1.0)$ $107724.5613(1.0)$ $1.4316 .4567(1.0$ $106079.1735(1.0)$ $106015 .+106(1.0)$ $106014.1123(0.3)$

106014.112310 .0

$105982.9734(1.0)$ $105945.7836(1.0)$

$123609.1589(1.0)$ $125670.7664(1.0)$ 121694.904911 .01 $123781.0059(1.0)$
$123679.158 \mathrm{~J}(1.0)$ $123679.158 \mathrm{~J}(1.0)$ $123685.4397(1.0)$

$123647.5443(1.3)$

$123603.7011(1.0)$

$141230.0299(1.0)$ $143613.3993(1.0)$ $139069.9503(1.0)$ $141433 ., 621(1.0)$ $141433 ., 621(1.0)$
$141340.3372(1.0)$ $141353.3762(1.0)$ $141353.8762(1.0)$
$141357.3575(1.0)$ $141357.3575(1.0)$ $141312.3373(1.0)$ $141312.3373(1.0)$
$141261.7115(1.0)$

$141201.4495(1.0)$

$141130.3054(0.0)$

$158835.9907(1.3)$ $161551.110)(1.0)$ $156440.8934(1.0)$ $159216.717 \mathrm{~J}(1.0)$ $158993.7141(1.0)$ $159033.3625(1.0)$ $159031.7017(1.0)$ $158978.5485(1.0)$

$159919.9775(1.0)$

$158851.5033(1.0)$

$159771.5993(1.0)$

$158679.1422(1.3)$

$176425.0058(1.3)$ $179484.2395(1.0)$

$175953.2573(1.0)$ $176653.3225(1.0)$ $176709.5325(1.0)$

$176706.7263(1.3)$
$176644.3133(1.0)$

$176578.2082(1$.

$176561.5027(1.0)$

$176412.295 ?(1.0)$

$176309.3005(1.0)$
$17673.5203(0.0009)$

$35345.1563(0.0317)$ $35913.8133(0.0017)$
$34777.5573(0.0317)$

$53013.0237(0.0325)$ $53869.4153(0.0324)$ $53019.8555(0.0321)$ $53012.5768(0.0021)$

$73675.237+(0.0031)$ $71823.4464(0.0030)$ $69551 .(593(0.0030)$ $70599.9453(0.0326)$ $70681.7497(0.0026)$ $70673.8281(0.0025)$
$79573.8063(0.0025)$ $88329.9151(0.0036)$ $89775.3737(0.0334)$
$86935.0173(0.0334)$ $88385.8550(0.0030)$ $83349.4781(0.0030)$ $39343.5751(0.0328)$ $83343.4990(0.0028)$ $88318.4963(0.0336)$ $88318.4963(0.0036)$

$105975.182+10.0039)$ $107724.6581(0.0336)$ $104316.4587(0.0036)$ $105079.0674(0.0032)$ $105015.4001(0.0032)$ $105614.2320(0.0330)$ $105013.9991(0.0330)$ $105932.8355(0.0338)$ $105982.8854(0.0338)$ $105945.7939(0.0054)$ $105945.7938(0.0054)$

$123609.1674(0.0043)$ $125670.7513(0.0037)$ $121694.8983(0.0037)$ $123780.9924(0.0333)$ $123679.1549(0.0134)$ $123685.9050(0.0030)$
$123585.4485(0.0330)$ $123585.4485(0.0330)$ $123647.6513(0.0340)$
$123647.6505(0.0040)$ $123603.6933(0.0355)$ $123603.6938(0.0055)$ $123551.3572(0.0072)$ $123551.357 ?(3.0372)$

$141230.0163(0.0349)$ $143613.0935(0.0037)$ $139069.8639(0.0037)$

$1+1473.0595(0.0034)$ $141340.3315(0.0035)$ $141358.9903(0.0029)$ $141357.9779(0.0330)$ $141312.2563(0.0039)$ $141312.8544(0.0039)$ $141251.7173(0.0353)$ $141251.7173(0.0053)$ $141201.4396(0.0365)$ 141201.4396(
$141130.7062(0.0366)$ $141130.7062(0.0086)$
$141130.7062(0.0036)$

$153835.8375(0.0350)$ $161551.1123(0.0038)$ $155440.8949(0.0039)$ $159216.7041(0.0038)$ $158938.7194(0.0039)$ $159033.3795(0.0029)$ $153031.7053(0.0030)$ $153978.5633(0.0039)$ $158978.5591(0.0039)$ $158919.8819(0.0052)$ $158919.8319(0.0352)$ $158851.4774(0.0359)$ $158851.4774(0.0359)$
$153851.4774(0.0059)$ $159771.5525(0.0071)$ $159771.5525(0.0071)$ $158771.5525(0.0371)$ $158679.1711(0.0128)$

$175425.0114(0.0079)$ $179484.2219(0.0343)$ $173907.5450(0.0048)$ $175953.2589(0.0347)$ $176653.8078(0.00491$ $176709.6057(0.0033)$ $176706.7369(0.0034)$ $175644.8371(3.0042)$ $175644.8269(0.0042)$ $175578.2053(0.0054)$ $176578.2053(0.0054)$ $175501.4650(0.0059)$ $175501.4650(0.00591$ $175412.2489(0.0355)$ 175412.24890 .00551 176412.243910 .00651 $176309.3342(0.0176)$

0.0512

0.590

0.000

0.0051

0.0051
0.0037

1.759

6.222
6.165

0.590

0.0123
-0.0018

$-3.0258$

$-0.0096$

c.0062

0.0079
0.0128

0.0055

$-3.0116$

0.0025

0.0110

0.0205

0.0138

$-0.0020$

- 0.0126

0.0679

0.0082
0.0129

3.537

8.019
7.905

21.236
21.235

5.894

10.415
10.225

23.594

23.593

45.709

45.709

8.841

13.409

13.125

26.540

48.656

48.656 
TABLE IX. OBSERVEO AN CALCULATEO FREQUENCIES OF DOSCO IN MHZ

(CONTINUEJ)

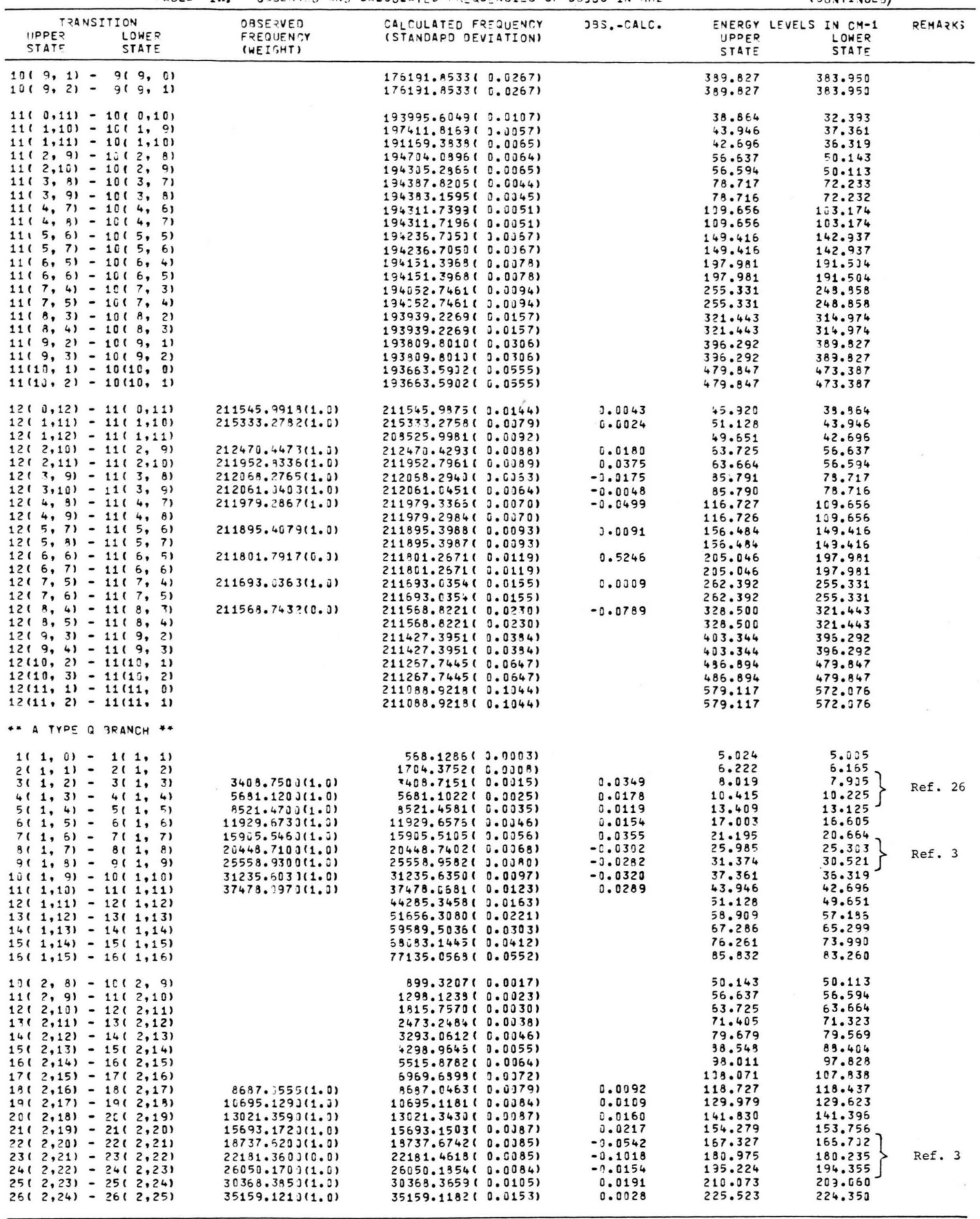




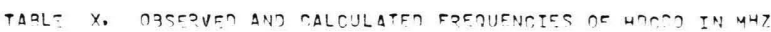

\begin{tabular}{|c|c|c|c|c|c|c|c|c|}
\hline \multicolumn{2}{|c|}{ TRANSITION } & \multirow{3}{*}{$\begin{array}{l}\text { ORSEOVET } \\
\text { FOEDUEN TY } \\
\text { (WETGHT) }\end{array}$} & \multirow{3}{*}{$\begin{array}{l}\text { CALCULATER FREDUENCY } \\
\text { (STANDAPI DEVIATTON) }\end{array}$} & \multirow{3}{*}{ OAS.-CALC. } & \multirow{3}{*}{$\begin{array}{l}\text { ENERGY } \\
\text { UEPFR } \\
\text { STATE }\end{array}$} & \multirow{3}{*}{\multicolumn{2}{|c|}{$\begin{array}{c}\text { LEVELS IV CYM-1 } \\
\text { LOWFO } \\
\text { STATE }\end{array}$}} & \multirow[t]{3}{*}{ PFMAFKS } \\
\hline UPPER & LOWC? & & & & & & & \\
\hline STATE & STATE & & & & & & & \\
\hline
\end{tabular}

** A TYDE ? RRANCH **

$1(0,1)-0(0,0)$

$2(0,2)-1(0,1)$ $2(1,1)-1(1,0)$ $2(1,2)-1(1,1)$

$3(0,3)-2(0,2)$ $3(1,2)-2(1,1)$ 311,3 $3(2,1)=2(2,0)$

$4(0,4)-3(0,3)$ $4(1,3)-311,21$ $4(1,4)=2(1,2)$ $4(2,2)=3(2,1)$ $4(2,3)=3(2,2)$ $4(3,1)=3(3,0)$

$5(0,5)-4(0,4)$ $5(1,4)-4(1,3)$ $5(1,5)=4(1,4)$ $5(2,4)=4(2,3)$ $5(3,2)=4(2,3)$ $5(3,2)=4(3,1)$ $5(4,1)-4(4,0)$ $5(4,2)=4(4,1)$

$6(0,5)-5(0,5)$ $6(1,5)-5(1,4)$
$6(1,6)-5(1,5)$ $6(1,6)-5(1,5)$
$6(2,4)-5(2,3)$ $6(2,4)=5(2,3)$
$5(2,5)=5(2,4)$ $5(2,5)=5(2,4)$
$6(3,3)=5(3,2)$ $6(3,4)=5(3,2)$ $6(4,2)=5(4,1)$ $5,1)=5(5,2)$ $6(5,2)=5(5,1)$

$7(0,7)=6(0,6)$ $7(1,7)-6(1,5)$ $7(2,5)=6(2,4)$ $7(2,5)=6(2,5)$ $7(3,4)-6(3,3)$ $7(3,5)-6(3,4)$ $7(4,3)-614,21$ $7(4,4)=6(4,3)$ $7(5,3)$ - $5(5,2)$ $7(6,1)=6(6,0)$

$8(0,8)-7(0,7)$ $8(1,7)=7(1,6)$ $2(1,8)=7(1,7)$ $8(2,7)=7(2,5)$ $2(3,5)-7(3,4)$ $8(3,6)-7(3,5)$ $8(4,4)-7(4,3)$ $8(4,5)=7(4,4)$ $8(5,3)-7(5,2)$ $8(5,4)=7(5,3)$ $8(6,2)=7(6,2)$ $8(6,3)=7(6,2)$ $8(7,2)=7(7,1)$

$9(0,9)-8(0,8)$ $9(1,8)=8(1,7)$ $9(1,9)=8(1,8)$
$9(2,7)=8(2,6)$ $9(2,8)-8(2,7)$ $9(3,6)=8(3,5)$ $9(3,7)-8(3,6)$ $9(4,5)=2(4,4)$ $9(4,5)=8(4,5)$ $9(5,4)-2(5,3)$ $9(5,4)-8(5,3)$ $9(5,3)-8(6,2)$ $9(7,2)-8(7,1)$ $918,11=$ $9(8,2)=8(3,1)$

$10(0,10)-9(0,9)$ $10(1,9)-9(1,2)$ $10(1,10)=0(1,9)$ $10(2,9)=9(2,8)$ $10(2,9)-9(2,8)$
$10(3,7)-9(3,6)$ $10(3,2)-9(3,7)$ $10(4,5)=9(4,5)$ $10(4,7)=9(4,5)$ $10(5,6)=9(5,5)$ $10(6,4)=9(6,3)$ $10(5,5)$ - 9(6, 4) $10(7,3)=9(7,2)$ $10(7,4)=9(7,3)$ $10(8,3)-9(3$, ?)

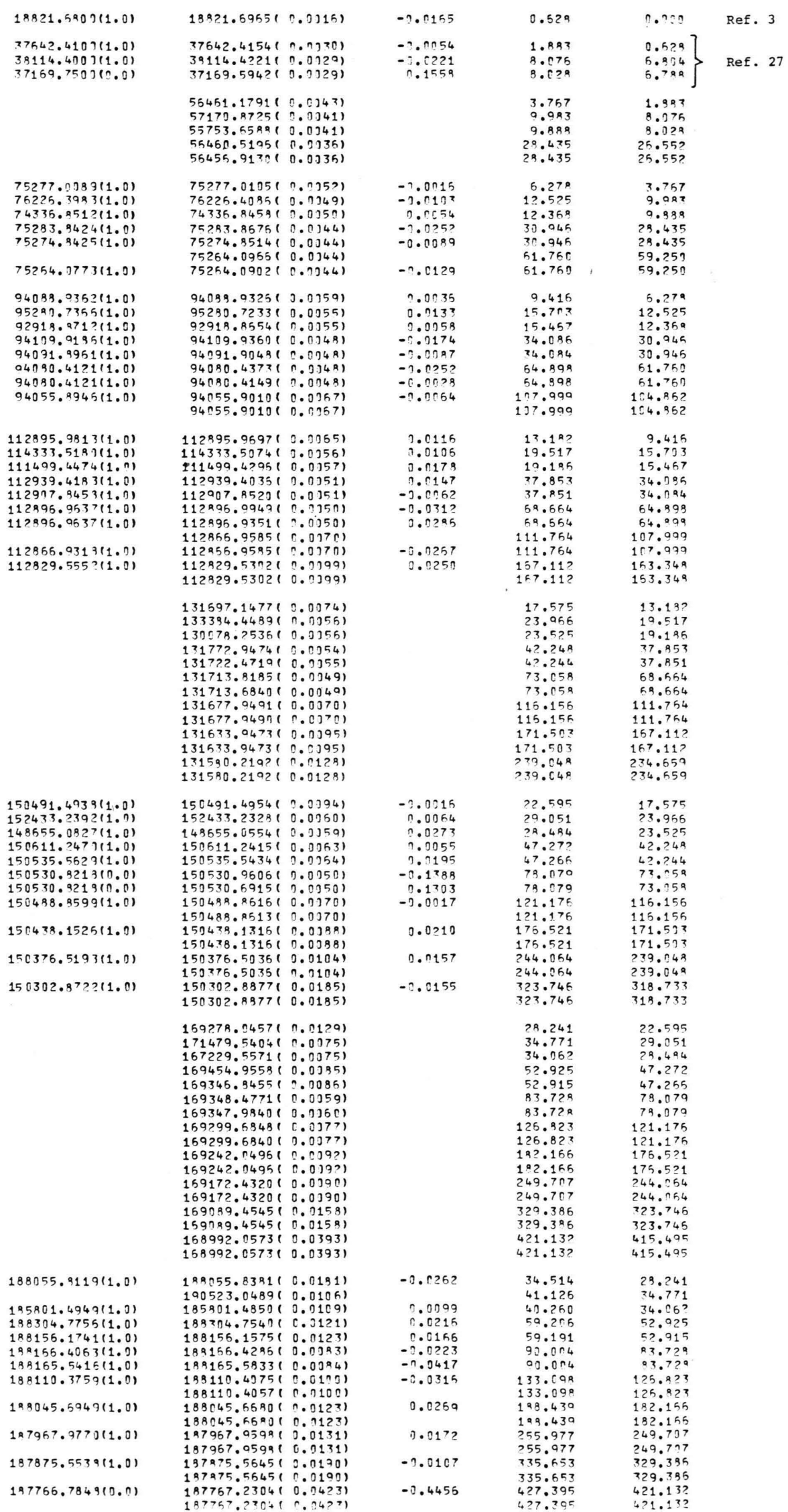




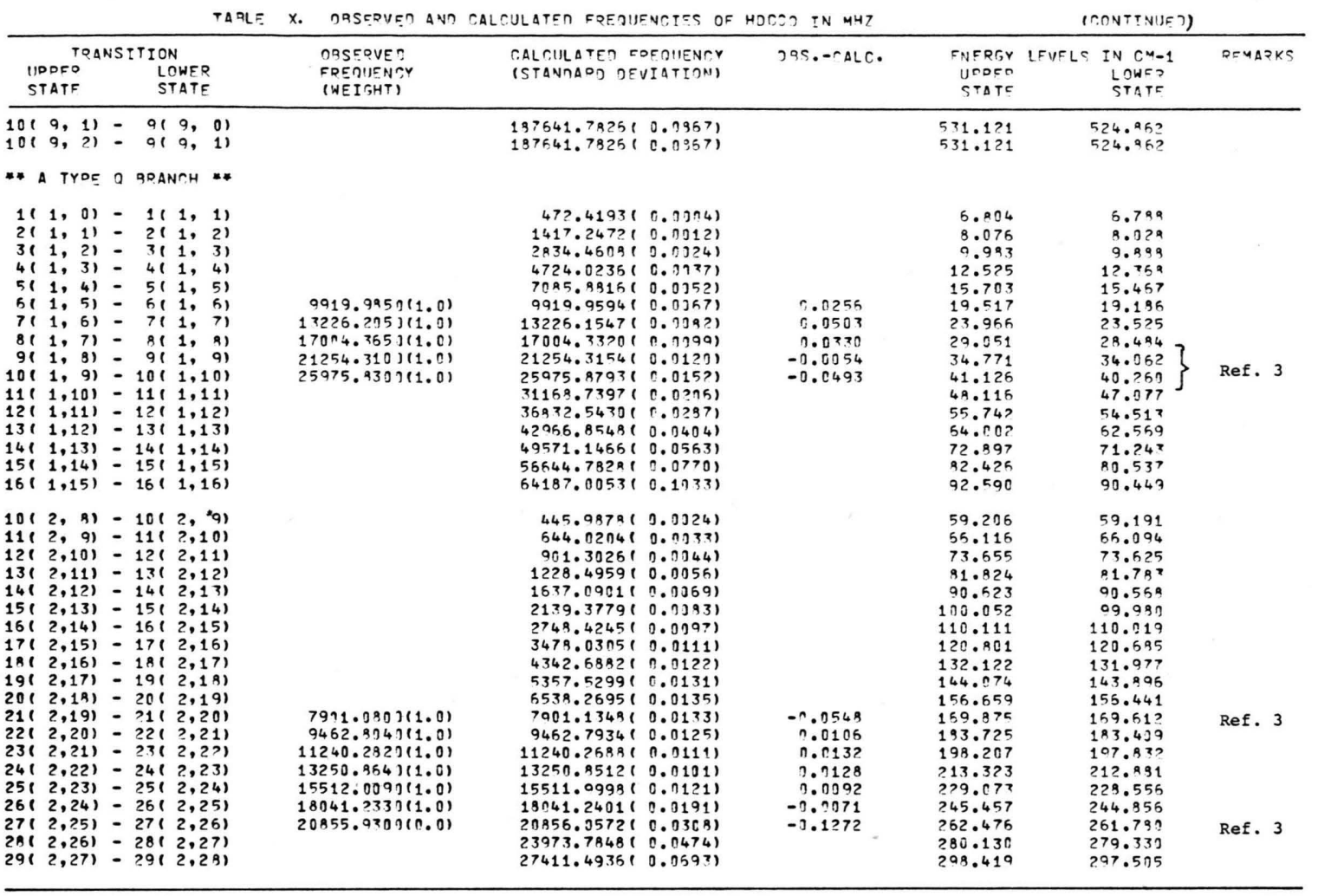

Tables VII and VIII show the variation of the derived parameters due to the different planarity conditions. These tables contain also the values of the quartic distortion constants $\hbar^{4} \tau_{a a b b}, \tau_{b b c c}^{\prime}$, $\tau_{c c a a}^{\prime}$ and $\hbar^{4} \tau_{a b a b}$ as calculated from normal-coordinate analysis ${ }^{11}$.

The errors of the determinable and derived rotational parameters were estimated on the basis of standard errors of the spectroscopic constants obtained from the least-squares process and are given in Tables I and II. The calculation of these errors was done via numerical propagation of errors, and was also checked by variance propagation formulae using zero co-variance assumptions.

\section{b) Discussion of Errors}

The problem whether there are systematic errors present in the results of our calculations was examined using residual analysis. The residual errors of the least-squares calculations were first reduced to "unit normal deviate" form (see Draper-Smith ${ }^{23}$ ) by dividing the residuals by their estimated error:

$$
s^{2}=\sum_{i}^{n} e_{i}^{2} /(n-p)
$$

where $n$ is the number of residual error data, while $(n-p)$ is the excess degrees of freedom of transitions fitted over the number of parameters in the Hamiltonian. The statistics of such a distribution should approach those of a normal distribution with zero average and unit standard deviation, provided there are no systematic errors present. The results of such an analysis are more or less in accord with the assumption of normality in the error distribution. The values of the standard deviation and the mean of the "unit normal deviate" residuals of ketene- $\mathrm{d}_{2}$ and ketene- $d_{1}$ are 0.9168 and -0.095 , and 0.9554 and 0.0292 , respectively.

Another test of residual correlations was made by examining the correlation among the residuals and the $K_{a}$ values. This test yielded coefficients of determination $r^{2}=0.134$ and 0.012 for ketene- $d_{1}$ and ketene- $\mathrm{d}_{2}$. A similar test of correlation among the residuals and their $J$ values yielded coefficients of determination: $r^{2}=0.042$ and 0.003 for ketene$d_{1}$ and ketene- $d_{2}$, respectively. These correlations are therefore insignificant.

As to the correlations found among constants obtained from the least-squares calculations, i. e. be- 
tween the spectroscopic constants in Watson's reduced representation the following were noted. The strongest correlations exist between the members of the following groups $(A, B, C),\left(A, \delta_{K}\right),\left(B, \delta_{K}\right)$, $\left(C, \delta_{K}\right)$ and $\left(\delta_{K}, \delta_{J}\right)$, while no significant correlations were found for the two sextic constants. The correlations among the rotational constants $A, B$ and $C$ are due to the fact that these three constants were refined separately. The correlations between $B$ and $\delta_{K}$, furthermore between $C$ and $\delta_{K}$ are analogous to the correlations found by Johns, Stone and Winnewisser ${ }^{6}$ for ketene- $\mathrm{h}_{2}$ between $(B-C)$ and $\delta_{K}$. This correlation was also pointed out by Sheridan ${ }^{10}$.

The results of the fitting procedure, the calculated and measured transitions, including some predicted transitions for ketene- $d_{2}$ and ketene- $d_{1}$ are found in Tables IX and X, respectively. As can be seen from the latter tables the standard error of prediction for both deuterated species is somewhat greater than for ketene- $\mathrm{h}_{2}{ }^{6}$. This is a consequence of the lack of high resolution infrared data on these molecular species.

Table XI. Comparison of inertial defect values in $\left[\mathrm{amu} \cdot \AA^{2}\right]$ from different representations of spectroscopic constants with values from normal-coordinate analysis (Ref. ${ }^{11}$ ).

\begin{tabular}{|c|c|c|c|}
\hline & $\begin{array}{l}\text { Reduced repr. } \\
\text { value }\end{array}$ & $\begin{array}{l}\text { Average of } \\
\text { derived const. }\end{array}$ & $\begin{array}{l}\text { Normal-coord. } \\
\text { value }\end{array}$ \\
\hline $\mathrm{CD}_{2} \mathrm{CO}$ & $0.114457(83)$ & $0.11386(10)$ & 0.11522 \\
\hline $\mathrm{CHDCO}$ & $0.10265(30)$ & $0.101993(35)$ & 0.10117 \\
\hline
\end{tabular}

1 B. Bak, E. S. Knudsen, E. Madsen, and J. Rastrup-Andersen, Phys. Rev. 79, 190 [1950].

2 H. R. Johnson and M. W. P. Strandberg, Phys. Rev. 82, 327a [1952].

3 H. R. Johnson and M. W. P. Strandberg, J. Chem. Phys. 20, 687 [1952].

4 C. B. Moore and G. C. Pimentel, J. Chem. Phys. 38, 2816 [1963].

5 A. P. Cox, L. F. Thomas, and J. Sheridan, Spectrochimica Acta 15, 542 [1959].

6 J. W. C. Johns, J. M. R. Stone, and G. Winnewisser, J. Mol. Spectrosc. 42, 523 [1972].

7 J. W. Williams and C. D. Hurd, J. Org. Chem. 5, 122 [1940].

8 M. Winnewisser, Z. Angew. Physik 30, 359 [1971].

9 M. Winnewisser and B. P. Winnewisser, Z. Naturforsch. 29 a, 633 [1974].

10 J. Sheridan, Advances in Molecular Spectroscopy, Fourth International Meeting on Molecular Spectroscopy, p. 139, Pergamon Press, New York 1962.

11 P. D. Mallinson and L. Nemes, J. Mol. Spectrosc., in press [1975].

12 S. R. Polo, Can. J. Phys. 35, 880 [1957].

13 J. W. C. Johns and W. B. Olson, J. Mol. Spectrosc. 39, 479 [1971].
The results obtained in this paper may be used to support the planar model of ketene, as the inertial defect values calculated from normal-coordinate analysis compare favourably with the various experimental inertial defect values. Table XI contains such a comparison which yields a satisfactory agreement. A similar conclusion may be derived from the value of the $\tau$ defect for ketene- $d_{2}$, although this quantity is more difficult to assess than the inertial defect values, and its relative error is greater in this work. The value of the $\tau$ defect was found to be undetermined by the present data for ketene- $d_{1}$. The origin of the $\tau$ defect is also the reason for the differences among the derived constants in Tables VII and VIII; they contain vibrational contributions due to zero-point vibrations.

\section{Acknowledgements}

One of the authors, L. N. would like to acknowledge the support of the present work, started at the Institut für Physikalische Chemie der Universität Kiel, by the Alexander von Humboldt Stiftung, Bonn-Bad Godesberg, in the form of a Dozentenstipendium during 1972/1973 and travel funds in 1975.

The authors express their gratitude for the help provided by Dr. Brenda P. Winnewisser and Dr. Koichi Yamada in the computational work and in the discussions about the least-squares procedure of the centrifugal distortion treatment.

14 J. K. G. Watson, J. Chem. Phys. 46, 1935 [1967].

15 J. M. Dowling, J. Mol. Spectrosc. 6, 550 [1961].

16 T. Oka and Y. Morino, J. Phys. Soc. Japan 16, 1235 [1961].

17 T. Oka and Y. Morino, J. Mol. Spectrosc. 11, 349 [1963].

18 W. H. Kirchhoff, J. Mol. Spectrosc. 41, 333 [1972].

19 R. L. Cook, G. Winnewisser, and D. C. Lindsey, J. Mol. Spectrosc. 46, 276 [1973].

20 R. L. Cook, F. C. De Lucia, and P. Helminger, J. Mol. Spectrosc. 53, 62 [1974].

${ }^{21}$ K. Yamada and M. Winnewisser, Z. Naturforsch. 30 a, 672 [1975].

22 R. L. Cook, F. C. De Lucia, and P. Helminger, J. Mol. Spectrosc. 41, 123 [1972].

23 N. Draper and H. Smith, Applied Regression Analysis, Wiley-Interscience, New York 1966.

24 E. R. Cohen and B. N. Taylor, J. Phys. Chem. Ref. Data 2, 663 [1973].

${ }^{25}$ K. V. L. N. Sastry and A. Guarnieri, Z. Naturforsch. 29 a, 1495 [1974].

26 W. H. Flygare and V. W. Weiss, J. Am. Chem. Soc. 37, 5317 [1965].

27 A. P. Cox and A. S. Esbitt, J. Chem. Phys. 38, 1636 [1963]. 